SLAC-PUB-8074

3/1999

\title{
THE GENERATION OF 400 MW RF PULSES AT X-BAND USING RESONANT DELAY LINES ${ }^{*}$
}

\author{
Sami G. Tantawi, Arnold E. Vlieks, and Rod J. Loewen \\ Stanford Linear Accelerator Center, Stanford University \\ P.O. Box 4349, MS 33, Stanford CA94309
}

\begin{abstract}
We present theory and experimental data for a resonant-delay-line pulse-compression system. The system is fed by two high power klystrons at X-band. The output power is four times bigger than the input power. The system produces flat-top output pulses. The system uses evacuated room-temperature copper delay lines as a means of storing energy. These lines achieved a quality factor greater than $4.3 \times 10^{5}$, with total losses due to external components measured at $4 \%$. We compare theory with experimental results.
\end{abstract}

Contributed to 1999 IEEE MTT-S International Microwave Symposium

Anaheim, California, June 13-19, 1999

\footnotetext{
*Work supported by Department of Energy contract DE-AC03-76SF00515.
} 


\title{
THE GENERATION OF 400 MW RF PULSES AT X-BAND USING RESONANT DELAY LINES
}

\author{
Sami G. Tantawi*, Arnold E. Vlieks, and Rod J. Loewen \\ Stanford Linear Accelerator Center, Stanford University \\ P.O. Box 4349, MS 33, Stanford CA94309
}

\begin{abstract}
We present theory and experimental data for a resonant-delay-line pulse-compression system. The system is fed by two high power klystrons at X-band. The output power is four times bigger than the input power. The system produces flat-top output pulses. The system uses evacuated room-temperature copper delay lines as a means of storing energy. These lines achieved a quality factor greater than $4.3 \times 10^{5}$, with total losses due to external components measured at $4 \%$. We compare theory with experimental results.
\end{abstract}

\section{INTRODUCTION}

Radio frequency pulse compression using resonant Delay Lines is proposed as a method for achieving the high-power flat rf pulse required to drive the Next Linear Collider (NLC) [1-2]. In order to achieve pulse compression, energy from an incoming rf pulse is stored in high $\mathrm{Q}$ resonant delay lines. While charging, energy that leaks out of the delay lines is, to great extent, canceled by the reflected incident rf. To discharge the lines, the phase of the incoming pulse is reversed so that the reflected signal from the inputs to the lines adds constructively with the emitted field from the stored energy in the lines for the duration of one round trip time of $\mathrm{rf}$ in the line.

The system suffers from two types of losses that reduce its efficiency: intrinsic losses and finite conductivity losses. By design, some of the input energy is immediately reflected at the delay line entrance during the charging phase. Additionally, after the phase reversal, the energy inside the lines is not discharged completely at the desired compressed pulse time period. Unfortunately, the coupling coefficient to the line that maximizes the energy storage makes the energy discharge from the line far from optimum. The system is designed for an optimum coupling coefficients at a specific compression ratio to maximize the output power. During the period of time the rf energy spends inside the storage line part of it is lost simply due to the finite quality factor of the lines. Similar losses occur from the finite conductivity of the components used to manipulate the input and output signals.

We describe the experimental procedures and the measurements performed on the high-power $\mathrm{X}$-band prototype built at the Stanford Linear Accelerator Center (SLAC).

\footnotetext{
* Also with the Communications and Electronics Department, Cairo University, Giza, Egypt
} 


\section{THEORY}

Consider a waveguide delay line terminated by a short circuit and coupled at its input with a coupling iris. The lossless scattering matrix representing the iris is unitary. At a certain reference plane the matrix takes the following form:

$$
\underline{\underline{S}}=\left(\begin{array}{cc}
-R_{0} & -j\left(1-R_{0}^{2}\right)^{1 / 2} \\
-j\left(1-R_{0}^{2}\right)^{1 / 2} & -R_{0}
\end{array}\right) .
$$

In writing Eq. (1) we assumed a symmetrical structure for the iris two port network. The forward and reflected fields around the iris are related as follows:

$$
\begin{gathered}
V_{1}^{-}=-R_{0} V_{1}^{+}-j\left(1-R_{0}^{2}\right)^{1 / 2} V, \\
V_{2}^{-}=-j\left(1-R_{0}^{2}\right)^{1 / 2} V_{1}^{+}-R_{0} V_{2}^{+} .
\end{gathered}
$$

With the exception of some phase change, the incoming signal $V_{2}^{+}$at time instant $t$ is the same as the outgoing signal $V_{2}^{-}$at time instant $t-\tau$; where $\tau$ is obviously the round trip delay through the line; i.e.

$$
V_{2}^{+}(t)=V_{2}^{-}(t-\tau) e^{-j 2 \beta l}
$$

where $\beta$ is the wave propagation constant within the delay line, and $l$ is the length of the line. Substituting from Eq. (4) into Eq. (3) we get

$V_{2}^{-}(t)=-j\left(1-R_{0}^{2}\right)^{1 / 2} V_{1}^{+}(t)-R_{0} V_{2}^{-}(t-\tau) e^{-j 2 \beta l}$.

During the charging phase we assume a constant input, i.e., $V_{1}^{+}(t)=V$ in which equals a constant value. We, also, assume that all the voltages are equal to zero at time $t<0$. Hence, substituting the solution of the difference equation (5) into Eq. (4) leads us to write

$V_{2}^{+}(i)=-j \frac{1-\left(-R_{0} e^{-j 2 \beta l}\right)^{i}}{1+R_{0} e^{-j 2 \beta l}}\left(1-R_{0}^{2}\right)^{1 / 2} e^{-j 2 \beta l}$ Vin.

In Eq. (6) $V_{2}^{+}(i)$ means the incoming wave in the time interval $i \tau \leq t<(i+1) \tau$ and $i \geq 0$. Substituting from Eq. (6) into Eq. (2) we get

$$
V_{1}^{-}(i)=-\operatorname{Vin}\left[R_{0}+\left(1-R_{0}^{2}\right) \frac{1-\left(-R_{0} e^{-j 2 \beta l}\right)^{i}}{1+R_{0} e^{-j 2 \beta l}} e^{-j 2 \beta l}\right]
$$

If the delay line has small losses ( $\beta$ has a small imaginary part), at resonance the term

$$
e^{-j 2 \beta l}=-p .
$$

where $p$ is a positive real number close to 1 . Eq. (7) becomes

$$
V_{1}^{-}(i)=-\operatorname{Vin}\left[R_{0}-\left(1-R_{0}^{2}\right) \frac{1-\left(R_{0} p\right)^{i}}{1-R_{0} p} p\right] \text {. }
$$

After the energy has been stored in the line one may dump part of the energy in a time interval $\tau$ by flipping the phase of the incoming signal just after a time interval $(n-1) \tau$, i.e.,

$V_{1}^{+}(t)= \begin{cases}\text { Vin } & 0 \leq t<(n-1) \tau \\ - \text { Vin } & (n-1) \tau \leq t<n \tau \\ 0 & \text { otherwise. }\end{cases}$

The output pulse level during the time interval $(n-1) \tau \leq t<n \tau$ can be calculated from Eq. (2) with the aid of Eq.(6). The result is

$V_{\text {out }}=V_{1}^{-}(n-1)=\operatorname{Vin}\left[R_{0}+\left(1-R_{0}^{2}\right) \frac{1-\left(R_{0} p\right)^{n-1}}{1-R_{0} p} p\right]$

Indeed, this is the essence of the pulse compression system.

The maximum power gain of the system is limited. Using Eq. (11), the power gain as $n \rightarrow \infty$ is

$$
\left.\left(\frac{V_{\text {out }}}{\text { Vin }}\right)^{2}\right|_{n \rightarrow \infty}=\left[R_{0}+\left(1-R_{0}^{2}\right) \frac{p}{1-R_{0} p}\right]
$$

which has a maximum value of

MaximumPowerGain $=\frac{17}{p^{2}}-8-\frac{12 \sqrt{2\left(1-p^{2}\right)}}{p^{2}}$

at 


$$
R_{0}=\frac{1}{p}-\frac{\sqrt{8\left(1-p^{2}\right)}}{4 p}
$$

Clearly the maximum power gain is limited to 9 as $p \rightarrow 1$ Furthermore, this maximum is greatly affected by the losses in the delay line; for example, the gain is limited to 7.46 if the line has a $1 \%$ round trip power losses.

\section{SYSTEM IMPLEMENTATION}

Figure 1 shows the pulse-compression system. It uses two 41.6-meter long cylindrical copper waveguides as delay lines, each 12.065 $\mathrm{cm}$ in diameter and operating in the $\mathrm{TE}_{01}$ mode. In theory, these over-moded delay lines can form a storage cavity with a quality factor $\mathrm{Q}>$ $1 \times 10^{6}$. A shorting plate, whose axial position is controllable to within $\pm 4 \mu \mathrm{m}$ by a stepper motor, coupling to the lines do not excite higher order modes provided that they are perfectly concentric with the waveguide axis.

A compact low-loss mode converter excites the $\mathrm{TE}_{01}$ mode just before each iris. These mode transducers, known as wrap-around mode converters, were developed specifically for this application. Both mode converters are connected to two arms of a high-power, overmoded, planer 3-dB hybrid. This hybrid is also designed specially for this application so that it can handle the super high power produced by this system. Therefore, the reflection from the lines exits through the third arm of this hybrid while the input to the lines is fed through the fourth arm. The distance from the irises to the center of the hybrid has been adjusted to within $\pm 13 \mu \mathrm{m}$ to maximize this transmission.

The iris reflection coefficient is was

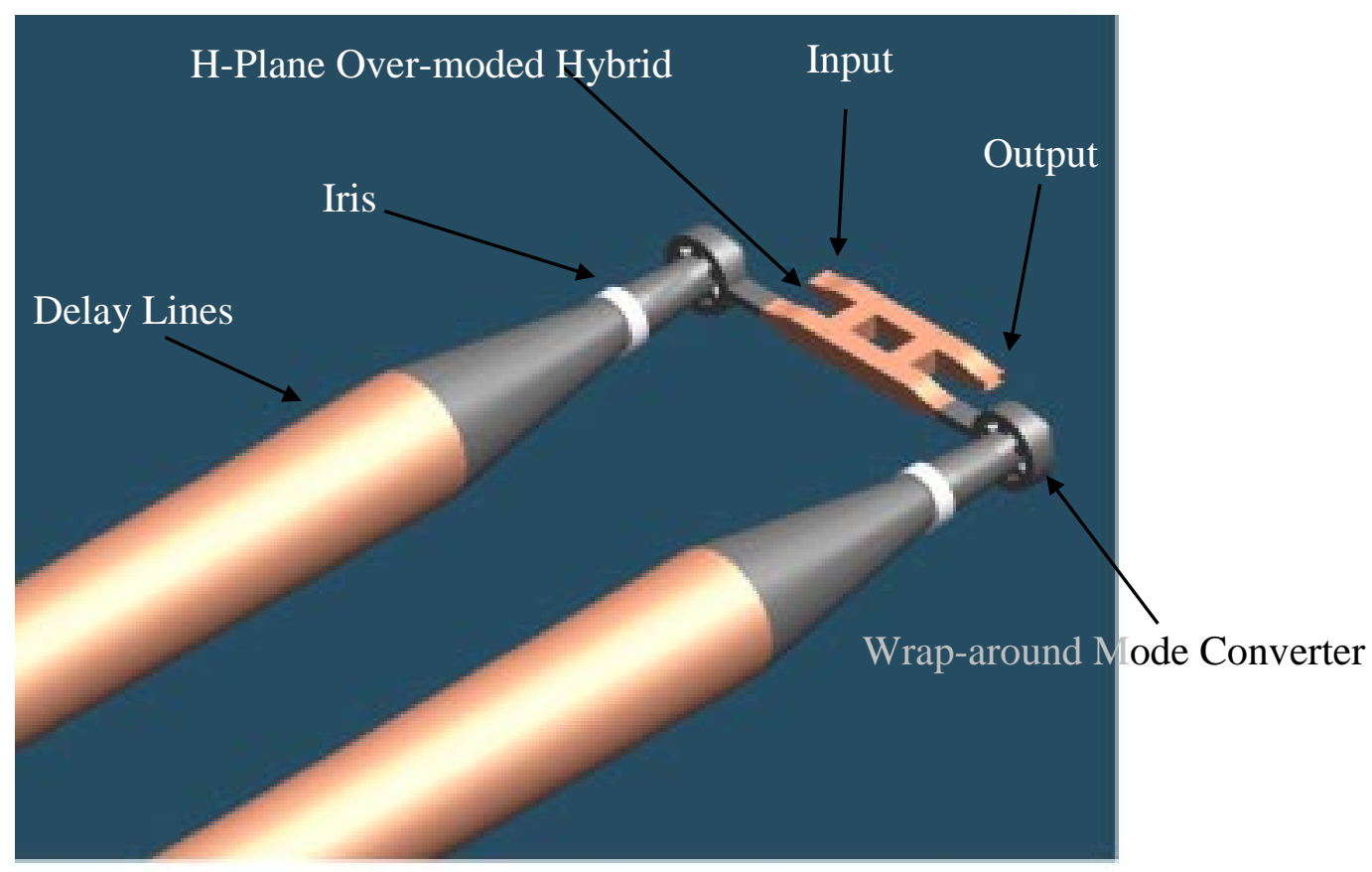

Figure 1 . Resonant Delay Line Pulse compression system

terminates each of the delay lines. The input of the line is tapered down to a $4.737 \mathrm{~cm}$ diameter waveguide at which the mode $\mathrm{TE}_{02}$ is cut-off; hence, the circular irises which determine the optimized for a compression ratio of 8 . 


\section{RESULTS}

The power gain was measured for a series of compression factors. A least-squares fitting of these measurements to theory is shown in Figure 3 . The round trip losses was found to be $2.45 \%$, indicating an intrinsic $Q$ for the lines of $4.3 \times 10^{5}$. The external losses are 4\%, and the iris reflection coefficient is 0.74 . The iris was designed using a mode matching code to have a reflection coefficient of 0.73 , the optimum value for a compression ratio of 8 .

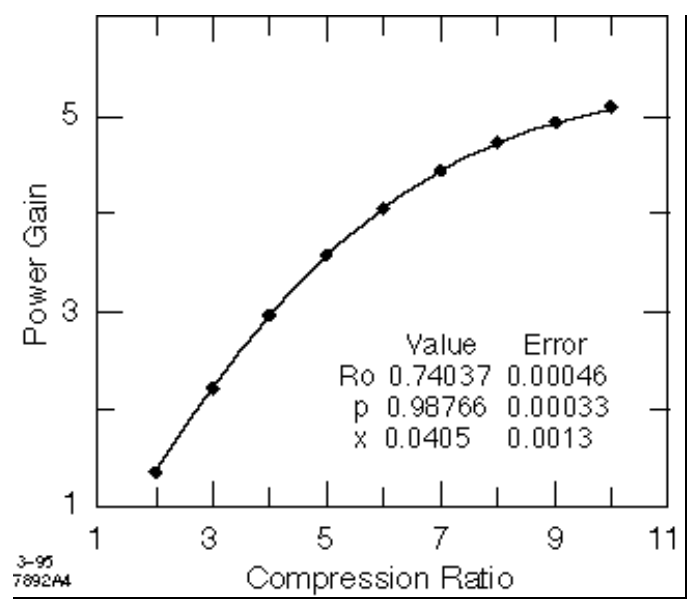

Figure 3. The points are measured power gains. The curve represents the least-squares fit.

This pulse compression is fed by two high power klystrons operating at $11.424 \mathrm{GHz}$. The each klystron is capable of producing $50 \mathrm{MW}$ of rf power. Hence, the maximum input power available for this compressor is a $100 \mathrm{MW}$. Figure 4. Shows the input and output of the compressor. The output power level is about $300 \mathrm{MW}$. The maximum power achieved by the system at the time of writing this paper. Going up in power is foreseen as the components get processed, and the vacuum pressure inside the system improves with time.

\section{CONCLUSION}

We presented an implementation of a super high power, resonant-delay-line pulse- compression

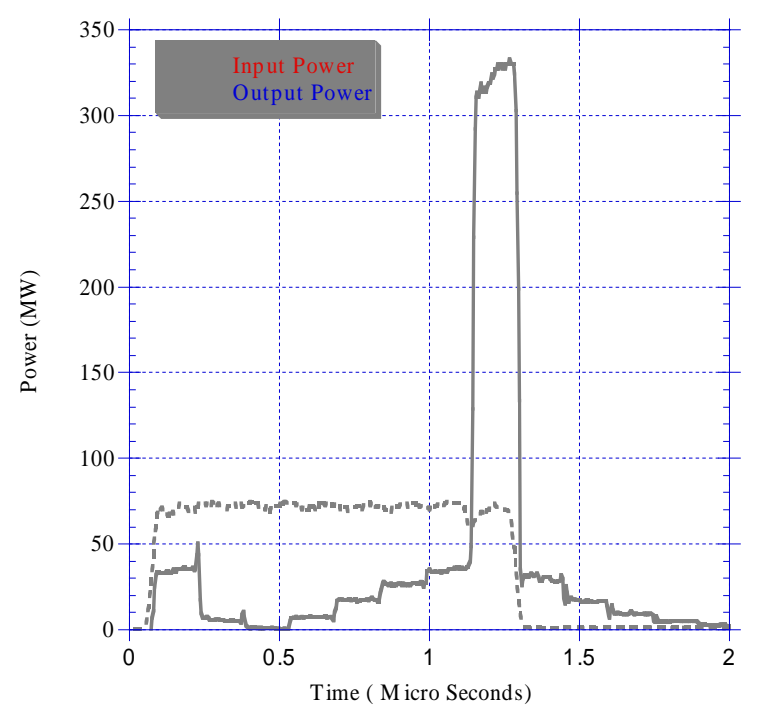

Figure 4. High-power pulse compressor output.

system. The output of the system agrees well with theory. The over-moded components and mode converters have proven to be capable of handling this level of rf power at X-band.

\section{ACKNOWLEDGMENT}

Work supported by Department of Energy contract DE-AC03-76SF00515

\section{REFERENCES}

[1] R. D. Ruth et al., "The Next Linear Collider Test Accelerator," Proceedings of the IEEE Particle Accelerator Conference, Washington DC, May 1993

[2] P. B. Wilson, Z. D. Farkas, and R. D. Ruth, "SLED II: A New Method of RF Pulse Compression," Linear Accl. Conf., Albuquerque, NM, September 1990; SLACPUB-5330 